\title{
DISTRIBUTED COMMUNICATIONS IN COLLISION CHANNELS WITH ERRORS
}

\author{
J.-T. LIM and S. M. MEERKOV† \\ Department of Electrical Engineering and Computer Science. The University of Michigan. Ann \\ Arbor, MI 48109-1109. U.S.A.
}

\begin{abstract}
Analysis of distributed communication networks in noisy collision channels is given. Both feedback and feedforward channel errors are considered. A finite number of buffered users is addressed. It is shown that channel errors lead to stabilization of unstable access control protocols. i.e. to elimination of the saturation phenomena and to stabilization of a network in a unique, globally asymptotically stable steady state with relatively high-performance characteristics. Thus channel noise, possibly introduced intentionally, could be viewed as a decentralized stabilizing controller.
\end{abstract}

\section{INTRODLCTION}

Professor Bellman viewed communications and control as important sources of problems in applied mathematics. The theory of communication networks was of particular interest to him. He wrote a number of papers and supervised several doctoral students in this area. Our research on communication networks in collision channels was initiated after extensive discussions with Professor Bellman on these and related topics. His guidance and advice during this work was an invaluable source of encouragement and support.

This paper is devoted to the problem of modelling and analysis of distributed communication networks in noisy collision channels, i.e. in collision channels with errors. The previous work in this area has been reported in [1]-[3] where the tree-type protocols[4] have been analyzed. It has been shown that the tree-type admission policies are extremely sensitive to errors in feedback channels [1, 2], unless special measures are taken[3].

In the present paper, a method of analysis applicable to any Markovian access control protocol is developed. Both feedback and feedforward channels errors are considered. Finite number of buffered users in slotted time environment is addressed. On the basis of the developed technique, it is shown that channel errors result in two new (as compared to noiseless situation) qualitative phenomena:

(i) "Continuous" degradation of the performance characteristics of Markovian access control protocols and, in addition, loss of information (packets) and erroneous information received by the users.

(ii) Destruction of the bistable behavior of the Markovian contention access control protocols, elimination of the saturation effects, and stabilization of the network in a unique, globally asymptotically stable steady state with relatively high delay-throughput characteristics.

To substantiate these claims and to present the developed method, in Sec. 2 below the channel model is introduced, in Sec. 3 the network model is constructed, in Sec. 4 an asymptotic method for analysis of slow-in-the-average Markov processes is described, in Sec. 5 an asymptotic approximation of the network equations is derived, and in Sec. 6 their analysis is presented. Sec. 7 gives the conclusions.

The material of this paper is based upon a technique developed in [5]-[7]. For the sake of brevity, the results of [5]-[7] are not repeated here, and the reader is referred to the cited articles for the explanation of the notations and results pertaining to the asymptotic state-space analysis of distributed communications in a noiseless collision channel.

\section{THE CHANNEL}

Analysis of distributed communications in noisy collision channel can always be reduced to analysis of the behavior of a set of users communicating with a single receiver across a noisy,

†To whom correspondence should be addressed. 
collision (feedforward) channel and having an acknowledgement signal sent by the receiver through a noisy, collisionless (feedback) channel. The feedback signals can be $K$-nary, where $K \in\{2,3\}$. It is assumed that

(a) the channel propagation delay is zerot;

(b) transmission of a packet across the feedforward channel requires a unit interval of time $(n-1, n], n=1,2, \ldots$;

(c) transmission of the feedback signal is instantaneous;

(d) the feedback signal at time $n$ is received by all users that have been active during the time slot $(n-1, n], n=1,2, \ldots$;

(e) feedback signals, received by all active users at time $n$, are identical.

Using assumptions (a)-(e), the model of a collision channel with errors is introduced as follows:

Let $\alpha_{i}(n), i=1, \ldots, K, n=1,2, \ldots$, denote the event occurred in the feedforward channel during time slot $(n-1, n)$. For the purposes of binary feedback, for example, $K=2$ and $\alpha_{1}(n)$ represents a successful transmission during $(n-1, n]$ and $\alpha_{2}(n)$ represents no successful transmission during $(n-1, n]$. Let $\beta_{i}(n), i=1, \ldots, K=1,2, \ldots$, denote an event recognized by the receiver as the event occurred in the feedforward channel during $(n-1$, $n$ ]. For the purposes of binary feedback, $K$ is again 2 , and $\beta_{1}$ and $\beta_{2}$ are associated with successful transmission and no successful transmission respectively. Let, finally, $X$ and $Y$ be random variables taking values $\left\{\alpha_{1}, \ldots, \alpha_{K}\right\}$ and $\left\{\beta_{1}, \ldots, \beta_{K}\right\}$, respectively. Then the model of the feedforward channel with errors can be given by a $K \times K$ constant, stochastic matrix $\mathbf{A}=\left(a_{i j}\right)$ such that

$$
a_{i j}=\operatorname{Prob}\left\{Y=\beta_{j}(n) \mid X=\alpha_{i}(n)\right\} .
$$

To characterize the feedback channel, introduce $\gamma_{i}(n)$ and $\delta_{i}(n), i=1, \ldots, K, n=$ $1,2, \ldots$, as the events transmitted and received in the feedback channel at time $n$, respectively. For binary feedback, $K=2$ and $\gamma_{1}(n)$ and $\gamma_{2}(n)$ represent positive acknowledgement and no acknowledgement sent by the receiver, whereas $\delta_{1}(n)$ and $\delta_{2}(n)$ represent the acknowledgement as recognized by the users, $\delta_{1}(n)$ as positive acknowledgement and $\delta_{2}(n)$ as no acknowledgement. Let $V$ and $W$ be random variables taking values $\left\{\gamma_{1}, \ldots, \gamma_{K}\right\}$ and $\left\{\delta_{1}, \ldots, \delta_{K}\right\}$, respectively. Then the model of the feedback channel with errors can be defined by the following $K \times K$ constant, stochastic matrix $\mathbf{B}=\left(b_{i j}\right)$ :

$$
b_{i j}=\operatorname{Prob}\left\{W=\delta_{j}(n) \mid V=\gamma_{i}(n)\right\} .
$$

Matrices A and B uniquely define the overall (round trip) channel errors according to

$$
C=\mathbf{A B}
$$

It might seem reasonable to choose $C$ as the initial description of a channel with errors. This is not true, however, since the "distribution" of round trip errors between the feedback and feedforward channels, rather than the round trip error itself, uniquely characterizes the network behavior (see Sec. 6).

\section{THE NETWORK}

Assume that

( $\alpha$ ) the network consists of $1<<M<x$ users communicating with a single receiver through a noisy collision channel and having acknowledgements sent by the receiver;

( $\beta$ ) every user has a buffer capable of storing $1 \leq N \leq x$ packets;

† More general channels having, for instance, nonzero propagation delays can be analyzed using the technique developed below. For the sake of simplicity, however, the analysis reported here is restricted to zero propagation delay channels. 
$(\gamma)$ the input traffic is a Bernoulli sequence with parameter $p_{a}$, i.e. during each time slot, $[n-1, n]$, every user generates a packet with probability $p_{a}$. Since at most one packet can be successfully transmitted across the feedforward channel during a time slot, to avoid trivialities it is assumed that

$$
M p_{a} \leq 1
$$

( $\delta$ ) if a packet is generated by a user having its buffer full, the newly generated packet is rejected; otherwise, it is stored in the first available (empty) cell of the buffer;

( $\epsilon)$ if a positive acknowledgement is received by a user at time $n$, the packet transmitted during $(n-1, n]$ is eliminated from the first cell of its buffer; otherwise it is stored and attempted for transmission at a later time slot, depending on an access control protocol;

$(\zeta)$ if a packet is eliminated from the first cell of a buffer, a packet stored in its $m$ th cell, $m=2, \ldots, N$, instantaneously "moves down" to be stored in cell $m-1$; thus a higher cell cannot be occupied if one of the lower cells is empty.

Let $h_{i}(n), i=1, \ldots, N, n=0,1, \ldots$ be the occupancy of the $i$ th layer of buffers (horizontal states, see [5]-[7]) at time $n$. The evolution of $h_{i}$ 's in the network defined by $(\alpha)-$ $(\zeta)$ can be characterized as follows:

$$
\begin{aligned}
h_{1}(n+1)= & h_{1}(n)+\zeta_{1}(n, n+1)-\xi(n+1)+\psi_{21}(n+1), \\
h_{2}(n+1)= & h_{2}(n)+\zeta_{2}(n, n+1)-\psi_{21}(n+1)+\psi_{32}(n+1), \\
& \cdots \\
h_{N}(n+1)= & h_{N}(n)+\zeta_{N}(n, n+1)-\psi_{N, N-1}(n+1), \\
& h_{N} \leq h_{N-1} \leq \cdots \leq h_{2} \leq h_{1} \leq M .
\end{aligned}
$$

The sequence $\zeta_{i}(n, n+1), n=0,1, \ldots$, represents the process of packet arrivals into the $i$ th layer of buffers, $i=1, \ldots, N$, during a time slot $[n, n+1]$. The event $\zeta_{i}(n$, $n+1)=l, l=0,1, \ldots, M$, means that $l$ packets have arrived into the $i$ th layer during $[n, n+1]$. Due to assumptions $(\gamma)$ and $(\zeta)$, the conditional probability distribution of $\zeta_{i}(n$, $n+1)$ is given by

$$
\begin{aligned}
P\left\{\zeta_{i}(n, n+1)=\right. & \left.l \mid h_{1}(n), \ldots, h_{N}(n)\right\}=\left(\begin{array}{c}
h_{i-1}-h_{i} \\
l
\end{array}\right) p_{a}^{l}\left(1-p_{a}\right)^{h_{i-1}-h_{i}-l} \\
& l=0,1, \ldots, h_{i-1}(n)-h_{i}(n), \quad i=1, \ldots, N \\
& n=0,1, \ldots, h_{0}=M .
\end{aligned}
$$

The sequence $\xi(n+1), n=0,1, \ldots$, describes the process of elimination of packets from the first layer of buffers. The event $\xi(n+1)=l, l=0,1, \ldots, h_{1}(n)$, means that $l$ users which had been active during $(n, n+1]$ have received, at time $n+1$, a positive acknowledgement, and, consequently, $l$ packets had been eliminated from the first buffer layer. Note that in a noiseless collision channel $l=0$ or 1 . The conditional probability distribution of $\xi(n+1)$ is defined by both the access control protocol and the round trip error matrix $C$. For Markovian protocols, the conditional probability that $l$ users have attempted the transmission during $(n, n+1]$ is a function of the states of the system, $h_{1}, \ldots, h_{N}$, at time $n$, say $F_{i}\left(h_{1}(n)\right.$, $\left.\ldots, h_{N}(n)\right)$. Consequently,

$$
\begin{aligned}
P\{\xi(n+1)= & \left.1 / h_{1}(n), \ldots, h_{N}(n)\right\}=c_{1} F_{1}\left(h_{1}(n), \ldots, h_{N}(n)\right), \\
P\{\xi(n+1)= & \left.l / h_{1}(n), \ldots, h_{N}(n)\right\}=c_{K_{1}} F_{l}\left(h_{1}(n), \ldots, h_{N}(n)\right), \\
& l=2,3, \ldots, h_{1}(n) .
\end{aligned}
$$


For example, in the case of Symmetric ALOHA whereby every busy user attempts, with probability $p$, a transmission during $(n, n+1)$, if the feedback is binary

$$
\begin{gathered}
P\{\xi(n+1)=1 / \cdots\}=c_{11} h_{1}(n) p(1-p)^{h_{1}(n)-1}, \\
P\left\{\xi(n+1)=l l \cdots=c_{21}\left(\begin{array}{c}
h_{1}(n) \\
l
\end{array}\right) p^{l}(1-p)^{h_{1}(n)-1},\right. \\
l=2, \ldots, h_{1}(n) .
\end{gathered}
$$

If the feedback is ternary ( $\alpha_{1}:$ successful transmission; $\alpha_{2}:$ no transmission; and $\alpha_{3}:$ collision), Symmetric ALOHA gives

$$
\begin{gathered}
P\{\xi(n+1)=1 / \cdots\}=c_{11} h_{1}(n) p(1-p)^{h_{1}(n)-1}, \\
P\{\xi(n+1)=l / \cdots\}=c_{31}\left(\begin{array}{c}
h_{1}(n) \\
l
\end{array}\right) p^{l}(1-p)^{h_{1}(n)-1}, \\
l=2, \ldots, h_{1}(n) .
\end{gathered}
$$

The sequence $\psi_{i, i-1}(n+1), n=0,1, \ldots, i=2,3, \ldots, N$ represents the transition of packets from buffer layer $i$ to buffer layer $i-1$, if the elimination of some packets from layer $i$ has occurred. The event $\psi_{i, i-1}(n+1)=l, l=0,1, \ldots, h_{1}(n)$ means that $l$ packets have moved at time $n+1$ from buffer layer $i$ to buffer layer $i-1$. The conditional probability distribution of $\psi_{i, i-1}(n+1)$ is

$$
\begin{aligned}
P\left\{\psi_{i, i-1}(n+1)=\right. & \left.1 / h_{1}(n), \ldots, h_{N}(n)\right\} \\
= & c_{11} F_{1}\left(h_{1}(n), \ldots, h_{N}(n)\right) d_{i}+c_{K 1} \\
& \times\left[\sum_{j=l}^{h_{1}(n)}\left(\begin{array}{l}
j \\
1
\end{array}\right) F_{j}\left(h_{1}(n), \ldots, h_{N}(n)\right) d_{i}\left(1-d_{i}\right)^{j-1}\right], \\
P\left\{\psi_{i, i-1}(n+1)=\right. & \left.l / h_{1}(n), \ldots, h_{N}(n)\right\} \\
= & c_{K !}\left[\sum_{j=l}^{h_{1}(n)}\left(\begin{array}{l}
j \\
l
\end{array}\right) F_{j}\left(h_{1}(n), \ldots, h_{N}(n)\right) d_{i}^{l}\left(1-d_{i}\right)^{j-l}\right], \\
& i=2, \ldots, N, \quad l=2, \ldots, h_{1}(n),
\end{aligned}
$$

where

$$
d_{i}=h_{i}(n) / h_{1}(n) .
$$

Equations (5) along with conditional probability distributions (6)-(9) define a model of a distributed communication network in a collision channel with error and any Markovian access control protocol. Analysis of this model is given below.

\section{THE METHOD}

Let $x_{i}(n)=h_{i}(n) / M$ be the normalized occupancy of the $i$ th layer of buffers. Then

$$
\begin{aligned}
x_{1}(n+1)= & x_{1}(n)+\frac{1}{M}\left[\zeta_{1}(n, n+1)-\xi(n+1)+\Psi_{21}(n+1)\right], \\
& \ldots \\
x_{N}(n+1)= & x_{N}(n)+\frac{1}{M}\left[\zeta_{N}(n, n+1)-\Psi_{N N-1}(n+1)\right], \\
& x \in[0,1 / M, \ldots, 1], \quad x_{N} \leq x_{N-1}, \ldots, \leq x_{1} \leq 1 .
\end{aligned}
$$


If $\zeta_{1}(n, n+1), \xi(n+1)$ and $\psi_{i, i-1}(n+1)$ take values of order 1 and if $M>>1$, Eqs. (10) represent a slow Markov walk process with $\epsilon=1 / M$. An asymptotic theory for analysis of such processes has been developed in [8] and [9] and applied to analysis of distributed communication networks in [10]-[12].

In a noisy collision channel, however, $\xi(n+1)$ and $\Psi_{i, i-1}(n+1)$ take values $0,1, \ldots$ $M=1 / \epsilon$. Under assumption $(\gamma)$, random variable $\zeta_{i}(n, n+1)$ also takes values $0,1, \ldots$, $1 / \epsilon$. Thus (10) is not a slow Markov walk, and the theory of [8] and [9] is not applicable. Using, however, the properties of the first- and second-order statistics of the random variables in brackets of (10), it is possible to generalize the theory of [8] so that it becomes applicable to the problem at hand.

Consider the equation

$$
\begin{aligned}
x(n+1)= & x(n)+\Phi(x(n), \xi(\mathrm{n})), \\
& x \in R^{N}, \quad \xi \in R^{S}, \Phi: R^{N} \times R^{S} \longrightarrow R^{N},
\end{aligned}
$$

where $\xi(n), n=0,1, \ldots$, is a sequence of independent random variables with the following conditional probability distribution:

$$
f(\xi(n) \mid x(n))=\left\{f_{1}\left(\xi_{1}(n) \mid x(n)\right), \ldots, f_{s}\left(\xi_{s}(n) \mid x(n)\right)\right\} .
$$

Assume that

$$
\begin{gathered}
E\{\Phi(x(n), \xi(n)) / x(n)\}=\epsilon \phi(x(n)), \\
\operatorname{var}\left\{\Phi_{i}(x(n), \xi(n)) / x(n)\right\}=\epsilon^{2} \kappa_{i}(n), \\
i=1, \ldots, N
\end{gathered}
$$

where $\phi(x)$ and $\kappa_{i}(x)$ are functions of the order 1 ,

$$
\begin{aligned}
\|\phi(x)\| & \leq R, \\
\left|\kappa_{i}(x)\right| & \leq R_{i}, \quad i=1, \ldots, N, \\
& \forall x \in Q \subset R^{N},
\end{aligned}
$$

and $R$ and $R_{i}$ are independent of $\epsilon$. Assume also that $\phi(x)$ and $\kappa(x)=\left\{\kappa_{1}(x), \ldots, \kappa_{N}(x)\right\}$ are Lipschitz, i.e. for $\forall x^{\prime} x^{\prime \prime} \in Q \subset R^{N}$,

$$
\begin{aligned}
& \left\|\phi\left(x^{\prime}\right)-\phi\left(x^{\prime \prime}\right)\right\| \leq r\left\|x^{\prime}-x^{\prime \prime}\right\|, \\
& \left\|\kappa\left(x^{\prime}\right)-\kappa\left(x^{\prime \prime}\right)\right\| \leq k\left\|x^{\prime}-x^{\prime \prime}\right\| .
\end{aligned}
$$

Since, as it follows from (13), $\Phi$ can take arbitrarily large values, however, with sufficiently small probabilities, the process defined by (11) wil be referred to as slow-in-the-average.

Along with (11) consider

$$
\begin{aligned}
y(n+1) & =y(n)+\phi(y(n)), \\
y \in R^{N}, \quad y^{0} & =y\left(n_{0}\right)=x\left(n_{0}\right)=x^{0} .
\end{aligned}
$$

\section{THEOREM 1}

Under the assumptions (13)-(14), for any $\sigma>0$ and $\tau>0$ there exist $\epsilon_{0}=\epsilon_{0}(\sigma)$ and $F=F(\tau)$ such that for any $0<\epsilon \leq \epsilon_{0}$,

$$
\begin{gathered}
P\left\{\left\|x\left(n, x^{0}, n_{0}\right)-y\left(n, x^{0}, n_{0}\right)\right\|<\sigma\right\}>1-\sigma F(\tau), \\
n \in\left[n_{0}, n_{0}+\tau / \epsilon\right],
\end{gathered}
$$

where $x\left(n, x^{0}, n_{0}\right), n \in\left[n_{0}, n_{0}+\tau / \epsilon\right]$ is a slow-in-the-average process defined by (11) and $y\left(n, x^{0}, n_{0}\right), n \in\left[n_{0}, n_{0}+\tau / \epsilon\right]$ is its asymptotic approximation defined by (15) which belongs, together with its $\sigma$-vicinity, to $Q$. 
THEOREM 2

Assume that all trajectories of (11) are bounded a.s. and the equilibrium of (15) is globally asymptotically stable. Then, under assumptions (13) and (14), for any $\delta>0$ there exists $\epsilon_{0}>0$ such that for all $0<\epsilon \leq \epsilon_{0}$

$$
\begin{gathered}
P\left\{\left\|x\left(n, x^{0}, n_{0}\right)-y\left(n, x^{0}, n_{0}\right)\right\|<\delta\right\}>1-\delta, \\
n \in\left[n_{0}, \infty\right) .
\end{gathered}
$$

The proofs of these theorems follow, with necessary modifications, the proofs of theorems in [8]; for the sake of brevity these proofs are not given here.

If the required sufficient conditions are satisfied, Theorem 2 constitutes the basis for the analysis described in this paper. If, however, only the weaker conditions of Theorem 1 are met, the local analysis, based on Theorem 1, should be augmented by the global analysis, based on the large deviations theory, in order to analyze the behavior of the network on $\left[n_{0}+\right.$ $\tau / \epsilon, \infty)$, i.e. in order to characterize the residence time in the domain of attraction of each locally asymptotically stable steady state (see [6] where such approach has been developed for an error free channel).

\section{ASYMPTOTIC APPROXIMATIONS}

To verify whether Eqs. (10) satisfy (13), it is sufficient to show that $(1 / M) \zeta_{i}(n, n+1)$ and $(1 / M) \xi_{i}(n+1)$ satisfy (13) [compare (7) and (8)].

For $\zeta_{i}(n, n+1)$, it is easy to see that

$$
\begin{aligned}
E\left\{\zeta_{i}(n, n+1) / x_{1}(n), \ldots, x_{N}(n)\right\} & =M p_{a}\left(x_{i-1}(n)-x_{i}(n)\right), \\
\operatorname{var}\left\{\zeta_{i}(n, n+1) / x_{1}(n), \ldots, x_{N}(n)\right\} & =M p_{a}\left(x_{i-1}(n)-x_{i}(n)\right)\left(1-p_{a}\right), \\
i & =1, \ldots, N .
\end{aligned}
$$

Since, as it is indicated in $(\gamma), M p_{a} \leq 1$ and since $1 / M=\epsilon$, the sequence $(1 / M) \zeta_{1}(n, n+1)$, $i=1, \ldots, N, n=0,1, \ldots$, meets $(13)$.

For $\xi(n+1)$, we obtain

$$
\begin{aligned}
E\left\{\xi(n+1) \mid x_{1}(n), \ldots,\right. & \left.x_{N}(n)\right\}=c_{11} F_{1}^{(M)}\left(x_{1}(n), \ldots, x_{N}(n)\right) \\
& +c_{X 1}\left[\sum_{i=2}^{M x_{1}(n)} l F_{l}^{M)}\left(x_{1}(n), \ldots, x_{N}(n)\right)\right] \triangleq G\left(x_{1}(n), \ldots, x_{N}(n), \mathrm{C}\right),
\end{aligned}
$$

$\operatorname{var}\left\{\xi(n+1) \mid x_{1}(n), \ldots, x_{N}(n)\right\}=c_{11} F_{1}^{(M)}\left(x_{1}(n), \ldots, x_{N}(n)\right)$

$$
+c_{K 1}\left[\sum_{l=2}^{M x_{1}(n)} l^{2} F_{l}^{(n)}\left(x_{1}(n), \ldots, x_{N}(n)\right)\right]-G^{2}\left(x_{1}(n), \ldots, x_{N}(n), \mathrm{C}\right) \text {, }
$$

where $F_{l}^{M}\left(x_{1}, \ldots, x_{N}\right) \triangleq F_{l}\left(M x_{1}, \ldots, M x_{N}\right)$. The superscript $(M)$ will be omitted below.

As it is seen from (17), the sequence $(1 / M) \xi(n+1), n=0,1, \ldots$, satisfies (13) if $F$ 's decay sufficiently fast. For example, in the case of Symmetric ALOHA, say, with binary feedback,

$$
\begin{aligned}
& \begin{aligned}
& E\left\{\xi(n+1) / x_{1}(n), \ldots, x_{v}(n)\right\}= c_{11} M p x_{1}(n)(1-p)^{M x_{1}(n)-1} \\
&+c_{21} M p x_{1}(n)\left[1-(1-p)^{M x_{1}(n)-1}\right], \\
& \operatorname{var}\left\{\xi(\mathbf{n}+1) / x_{1}(n), \ldots, x_{v}(n)\right\}=E\{\xi(n+1)\}[1-E\{\xi(n+1)\}] \\
&+
\end{aligned} \\
& +c_{21}\left\{M p x_{1}(n)\left(M p x_{1}(n)-p\right)\right\} .
\end{aligned}
$$

Since $M p$ is of the order 1 (see [7]), Symmetric ALOHA with binary feedback satisfies (13). This is true for $K$-nary feedback as well. 
As far as condition (14) is concerned, it follows from (16), (17) and (8) that it is satisfied if $F_{/}\left(x_{1}, \ldots, x_{N}\right), l=1, \ldots, M$ are Lipschitz functions. This is certainly true for the Symmetric ALOHA system [see (17a)].

Thus all the conditions of Theorem 1 are met, and the asymptotic approximation (15) for the case of Eq. (10) can be written as follows:

$$
\begin{gathered}
y_{1}(n+1)=y_{1}(n)+\epsilon\left[\left(1-y_{1}(n)\right) M p_{a}-\frac{y_{1}(n)-y_{2}(n)}{y_{1}(n)} G\left(y_{1}(n), \ldots, y_{N}(n), \mathbf{C}\right)\right], \\
y_{2}(n+1)=y_{2}(n)+\epsilon\left[\left(y_{1}(n)-y_{2}(n)\right) M p_{a}-\frac{y_{2}(n)-y_{3}(n)}{y_{1}(n)} G\left(y_{1}(n), \ldots, y_{N}(n), \mathbf{C}\right)\right], \\
\ldots \\
y_{N}(n+1)=y_{N}(n)+\epsilon\left[\left(y_{N-1}(n)-y_{N}(n)\right) M p_{a}-\frac{y_{N}(n)}{y_{1}(n)} G\left(y_{1}(n), \ldots, y_{N}(n), \mathbf{C}\right)\right],
\end{gathered}
$$

where $G(y, \mathbf{C})$ is defined in (17).

In the case of Symmetric ALOHA, for instance, Eq. (18) becomes

$$
\begin{aligned}
y_{1}(n+1)= & y_{1}(n)+\epsilon\left\{\left(1-y_{1}(n)\right) M p_{a}-\frac{y_{1}(n)-y_{2}(n)}{y_{1}(n)} M_{p y_{1}}(n)\left[c_{11}(1-p)^{M y_{1}(n)-1}\right.\right. \\
& \left.\left.+c_{K 1}\left(1-(1-p)^{M y_{1}(n)-1}\right)\right]\right\} \\
& \cdots \\
y_{N}(n+1)= & y_{N}(n)+\epsilon\left\{\left(y_{N-1}(n)-y_{N}(n)\right) M p_{a}-\frac{y_{N}(n)}{y_{1}(n)} M p y_{1}(n)\left[c_{11}(1-p)^{M_{N_{1}}(n)-1}\right.\right. \\
& \left.\left.+c_{K 1}\left(1-(1-p)^{M_{y_{1}}(n)-1}\right)\right]\right\} .
\end{aligned}
$$

It follows from Theorem 1 that the solutions of $(10)$ define, with large probability, the behavior of (18) on a large, but finite time intervals. If, in addition, (18) has a globally asymptotically stable equilibrium point, this correspondence takes place for all $n \in\left[n_{0}, \infty\right]$ (Theorem 2).

\section{ANALYSIS}

It has been shown in [5]-[7] that the steady states of a distributed communication network, described by asymptotic approximation (18) (with $\mathbf{C}=I$ ), are defined by the intersections of the load and transmission lines. The load line does not depend on the fact that the channel is noisy and is defined by the steady state occupancy of the layers of buffers, $y_{i s}, i=1, \ldots$, $N$. It was shown that

$$
y_{i s}=y_{1 s}^{i}, \quad \text { if } N=\infty \text {, }
$$

and

$$
y_{i s}=y_{1 s} \frac{y_{(i-1) s}-y_{N s}}{1-y_{N s}}, \text { if } N<x
$$

Eliminating the intermediate variables, (20) can be rewritten as

$$
y_{i s}=y_{i s}^{(N)}\left(y_{1 s}\right),
$$


where $y_{i s}^{(*)}(\cdot)$ is a monotonically increasing algebraic function defined by parameter $N$. For instance, if $N=2$.

$$
y_{2 s}=y_{s}\left(y_{1 s}\right)=\frac{y_{15}+1}{2}-\sqrt{\frac{1+2 y_{1 s}-3 y_{1 s}^{2}}{4}} .
$$

In terms of $y_{i s}^{(v)}\left(y_{1 s}\right)$, the load line is defined by

$$
L\left(y_{1 s}\right) \doteq\left[1-y_{N s}^{(W)}\left(y_{1 s}\right)\right] M p_{a}
$$

The transmission line in a noiseless channel is defined as

$$
T L \triangleq F_{1}\left(y_{1 s}, y_{2 s}^{(N)}\left(y_{1 s}\right), \ldots, y_{N_{s}}^{\left(W_{i}\right)}\left(y_{1 s}\right), I\right) \triangleq \dot{F}_{1}^{(N)}\left(y_{1 s}\right)
$$

[We omit below the superscript $(N)$.] The intersections of (21) and (22) are the steady states of a network. A steady state is asymptotically stable, if in the point of intersection $\partial(T L) /$ $\partial y_{1 s}>\partial L / \partial y_{1 s}$; the opposite inequality implies instability. If $\tilde{F}_{1}\left(y_{1 s}\right)$ has a unique maximum on $[0,1]$, the network has three equilibria, the first and the third one are asymptotically stable and the second one is unstable. Unfortunately, the throughput in an asymptotically stable steady state can be very low (typically, in the third equilibrium). The effect of transition from the first to the third steady state is termed the saturation phenomenon and is interpreted as loss of throughput in the network (see [5-7] for analysis of the noiseless situation).

In a collision channel with errors the transmission line (22) is substituted, as it follows from (18), by a function describing the packet elimination $(P E)$ process:

$$
P E \triangleq G\left(y_{1 s}, y_{2 s}^{(N)}\left(y_{1 s}\right), \ldots, y_{N_{s}}^{(N)}\left(y_{1 s}\right) . \mathbf{C}\right) \triangleq \bar{G}^{(N)}\left(y_{1 s}, \mathbf{C}\right)
$$

[The superscript $(N)$ again will be omitted below.] For instance, in the case of Symmetric ALOHA for any $N$,

$$
\tilde{G}\left(\mathrm{y}_{1 s}, \mathbf{C}\right)=M p y_{1 s}\left[c_{11}(1-p)^{M y_{1 s}-1}+c_{K 1}\left(1-(1-p)^{M y_{1 s}-1}\right)\right] .
$$

Since the properties of $T L$ and $P E$, defined by (22) and (23), respectively, are different, the steady state behaviors of a network in noisy and noise free channels are also different. To quantify these differences, introduce

$$
\text { Hypothesis 1. Function } \bar{Z}_{1}(x)=\sum_{l=1}^{M x} l F_{l}\left(x, x_{2 s}^{(N)}(x), \ldots, x_{N s}^{(N)}(x)\right) \triangleq \sum_{l=1}^{M x} l \tilde{F}_{l}(x), x \in
$$

$[0,1 / M, \ldots, 1]$ is such that $\hat{Z}_{1}(y), y \in[0,1]$ is continuous and takes values of order $1<<M$.

Hypothesis 2. Both $\bar{Z}_{1}(y)$ and $\bar{Z}_{2}(y)=\tilde{Z}_{1}(y)-\tilde{F}_{1}(y), y \in[0,1]$, are strictly monotonically increasing.

Hypothesis A. Function $\bar{F}_{1}(y)$ is strictly monotonically increasing on $[0,1]$, i.e. in an error free channel the network has a unique equilibrium point and no saturation phenomena occurs.

Hypothesis B. Function $\tilde{F}_{1}(y)$ has a maximum on $(0,1)$, i.e. in an error free channel the network, with $N$ sufficiently large, has at least three equilibrium points and, consequently, exhibits a saturation phenomenon.

Hypotheses 1 and 2 are met by most Markovian access control protocols, such as ALOHA, CSMA, GRA, Random TDMA and others. For Symmetric ALOHA, for instance, $\bar{Z}_{1}(y)=M p y$ and $\bar{Z}_{2}(y)=M p y-M p y(1-p)^{M_{y}-1}$, both continuous, monotonically increasing functions of the order 1. Analogous expressions are true for other protocols.

Hypothesis A holds for such protocols as Random TDMA or Reservation ALOHA. Hypothesis B holds for ALOHA. CSMA, GRA and other contention protocols. In all of them, the conditional probability of successful transmission has a unique maximum on $(0,1)$.

An average steady state number of packets eliminated from the user's buffers during a 
time slot due to errors in the channel will be referred to as the lost information (LI). An average steady state number of packets recognized erroneously as successfully transmitted information will be referred to as the erroneous information (EI). An average steady state number of packets successfully transmitted across the channel during a time slot we refer to. as usually, as the throughput (TR). Obviously, in a noise free channel, $\mathrm{LI}=0$ and $\mathrm{EI}=0$.

\section{THEOREM A}

Assume that a given access control protocol satisfies Hypotheses 1,2 and $\mathrm{A}$. Then there exists a positive integer $M_{0}^{\dagger}$ such that a network, defined by $(\alpha)-(\zeta)$, with $M \geq M_{0}$ users utilizing the given protocol, in a channel defined by (a)-(e), with any round trip matrix $\mathbf{C}$ has a unique, globally asymptotically stable steady state $y_{t s}^{*} \ldots, y_{\mathrm{N}}^{*}$. In this steady state

$$
\begin{gathered}
\mathrm{TR}=a_{11} \tilde{F}_{1}\left(y_{1 s}^{*}\right), \\
\mathrm{LI}=\dot{G}\left(y_{s s}^{*}, \mathrm{C}\right)-c_{11} \tilde{F}_{1}\left(y_{1 s}^{*}\right), \\
\mathrm{EI}=\sum_{i=2}^{K} a_{i 1}\left[1-\dot{F}_{1}\left(\mathrm{y}_{1 s}^{*}\right)\right],
\end{gathered}
$$

where $a_{i 1}$ and $c_{11}$ are elements of $\mathbf{A}$ and $\mathbf{C}$, respectively, and $y_{1 s}^{*}$ is the point of intersection of (21) and (23).

Proof. If Hypothesis 1 holds, the conditions of Theorem 1 are satisfied and, therefore, there exist $M_{0}\left(\epsilon_{0}=1 / M_{0}\right)$ such that for all $M \geq M_{0}\left(\epsilon \leq \epsilon_{0}\right)$ the network defined by $(\alpha)-(\zeta)$ in a channel defined by (a)-(e) is indeed described by Eq. (18), associated with the original system (10) through the inequality of Theorem 1 . The steady states of (18) are defined by the intersections of (21) and (23).

Due to Hypothesis $\mathrm{A}$, with $\mathbf{C}=I$, the system has one steady state. Due to Hypothesis 2 , this situation remains true for any $\mathrm{C}$. Indeed

$$
G^{\prime}\left(y_{1 s}, \mathbf{C}\right)=c_{11} F_{1}^{\prime}\left(y_{1 s}\right)+c_{K 1} Z_{2}^{\prime}\left(y_{1 s}\right)
$$

which is positive, due to Hypotheses 2 and $\mathrm{A}$, for all $y_{\mathrm{ls}} \in[0,1]$.

By the indirect Liapunov method, it is easy to show that this steady state is globally asymptotically stable (see [5] for an analogous calculation). Thus Theorem 2 holds. Consequently, (18) describes the behavior of the network (10) with large probability on $\left[n_{0}, x\right)$, and therefore, TR, LI and EI can be defined as the throughput, lost information and erroneous information in the point of intersection of (21) and (23). It follows from the construction, that TR, LI and EI are given by (24)-(26). Q.E.D.

Consider, for example, the Random TDMA. Here for any $N$.

$$
\begin{aligned}
& \bar{F}_{1}\left(y_{1 s}\right)=y_{1 s}, \\
& \bar{F}_{(}\left(y_{1 s}\right)=0, \quad l=2, \ldots, M .
\end{aligned}
$$

Obviously, Hypotheses 1, 2 and A hold. Therefore,

$$
\begin{aligned}
\mathrm{TR} & =a_{11} y_{i s}^{*}, \\
\mathrm{LI} & =0, \\
\mathrm{EI} & =\left(\sum_{i=2}^{\kappa} a_{i 1}\right)\left[1-y_{i s}^{*}\right] .
\end{aligned}
$$

\section{THEOREM B}

Assume that a given access control protocol satisfies Hypotheses 1, 2 and B. Then there exist a positive integer $M_{0}$ and a positive constant $c_{0}$ such that a network, defined by $(\alpha)-(\zeta)$,

$\div M$ has been numerically estimated in [5] and [7] to be 50 . 
with $M \geq M_{0}$ users utilizing the given protocol, in a channel, defined by (a)-(e) with a round trip error matrix $\mathbf{C}$ satisfying the inequality

$$
c_{K 1} \geq c_{0},
$$

has a unique, globally asymptotically stable steady state $y_{t s}^{*}, \ldots, y_{v s}^{*}$. The throughput, the lost information and the erroneous information in this steady state are defined by (24)-(26), respectively.

Proof. The proof of this theorem repeats the first and the third paragraphs of the proof of Theorem $\mathrm{A}$. The second paragraph is substituted by the following:

Due to Hypothesis $\mathrm{B}$, and since (21) is a monotonically decreasing function, with $\mathbf{C}=I$, the network has, at least, three steady states. Let us show that under Hypothesis 2, there exist $c_{0}$ such that in a channel with $\mathbf{C}$ satisfying (27), the network has a unique steady state.

Indeed, the steady state is unique if $\bar{G}^{\prime}\left(y_{1 s}, \mathbf{C}\right)>0, \forall y \in[0,1]$. At a point $y_{1 s}$ where $\tilde{F}_{1}^{\prime}\left(y_{1 s}\right)>0$,

$$
\tilde{G}^{\prime}=c_{11} \tilde{F}_{1}^{\prime}+c_{K 1} \bar{Z}_{2}^{\prime}>0 \text {. }
$$

due to Hypothesis 2. At a point $y_{1 s}$ where $\tilde{F}_{1}^{\prime}\left(y_{1 s}\right)<0$,

$$
\tilde{G}^{\prime}=\left(c_{11}-c_{K 1}\right) \dot{F}_{1}^{\prime}+c_{K 1} \dot{Z}_{1}^{\prime}>0,
$$

if

$$
c_{K 1}>D c_{11} \text {, }
$$

where

$$
D \triangleq \sup _{\omega \in \Omega} \frac{\left|\bar{F}_{1}^{\prime}\left(y_{1 s}\right)\right|}{\dot{Z}_{2}^{\prime}\left(y_{15}\right)}
$$

and the Borel set $\Omega \triangleq\left\{y_{t s} \in[0,1]: \bar{F}^{\prime}\left(y_{t s}\right)<0\right\}$. Due to Hypothesis $2, D<1$. Consequently, the network has a unique steady state if

$$
D c_{11} \triangleq c_{0} \leq c_{K 1}
$$

Consider, for example, the Symmetric ALOHA system. Here functions $\bar{F}_{1}$ and $\bar{Z}_{i}, i=1$, 2, can be represented, for large $M$, as

$$
\begin{aligned}
& \tilde{F}_{1}\left(y_{1 s}\right)=G \exp \{-G\}, \\
& \tilde{Z}_{1}\left(y_{1 s}\right)=G, \\
& \dot{Z}_{2}\left(y_{1 s}\right)=G[1-\exp \{-G\}],
\end{aligned}
$$

where $G=M y_{1 s} p$. Consequently,

$$
D=\sup _{\omega \in \Omega} \frac{|\exp \{-G\}[1-G]|}{1-\exp \{-G\}[1-G]}=\frac{e^{-2}}{1+e^{-2}}=0.119 .
$$

Therefore, if in a binary feedback situation

$$
0.119 c_{11} \leq c_{21} \leq 1,
$$

the Symmetric ALOHA system has a unique, globally asymptotically stable steady state. The maximum stable throughput achieved in the system is

$$
\mathrm{TR}=a_{11} M y_{1 s} p(1-p)^{\mathrm{M}_{1,}-1} \cong a_{11} G \exp \{-G\},
$$


and

$$
\begin{aligned}
& \mathrm{LI}=0.119 c_{11} M y_{1 s} p\left[1-(1-p)^{M y_{15}-1}\right] \cong 0.119 c_{11} G[1-\exp \{-G\}], \\
& \mathrm{EI}=a_{21}\left[1-M y_{1 s} p(1-p)^{M y_{15}-1}\right] \cong a_{21}[1-G \exp \{-G\}] .
\end{aligned}
$$

Obviously, if $a_{11}$ is close to 1 , i.e. if probability of an error in the feedforward channel is small, the degradation in the throughput, as compared with noiseless, unstable situation, is insignificant. However, to achieve the stability, the lost information cannot be less than $0.119 c_{11} G[1-\exp \{-G\}]$.

Remark 1. If the channel noise is not strong enough to satisfy (27), the bistable behavior of network satisfying Hypothesis B will persist. However, it is possible to show that the residence time in the domain of attraction of the low throughput steady state will decrease. This conclusion can be arrived at using the large deviation technique described in [6].

Remark 2. Using Theorems A and B and the Little's formula, we conclude that the steady state time delay (TD) in a noisy channel is given by

$$
\mathrm{TD}=\frac{M \sum_{i=1}^{N} y_{i s}^{(N)}\left(y_{i s}^{*}\right)}{a_{11} \bar{F}_{1}\left(y_{1 s}^{*}\right)} .
$$

Remark 3. It is reasonable to assume that in most situations $a_{i 1}=0, i=2, \ldots, K$, i.e., the event "no successful transmission" cannot be recognized as a successful transmission in the feedforward channel. In this situation, $\mathrm{EI}=0$. If no errors at all occur in the feedforward channel, $A=I$, the stability condition (27) becomes

$$
b_{K 1} \geq c_{0} \text {, }
$$

and

$$
\mathrm{TR}=\tilde{F}_{1}\left(y_{1 s}^{*}\right)
$$

Consequently, errors in the feedback channel, satisfying (30), cause no degradation of the throughput, create no erroneous information and stabilize the channel.

\section{CONCLUSIONS}

In this paper, analysis of Markovian distributed communication networks in collision channels is given. It is shown that errors in the feedfoward channel lead to degradation in the throughput and time delay of the network as well as to erroneous information. Both feedback and feedforward channels errors lead to a loss of information (packets). These losses and erroneous information could be countermeasured by coding techniques.

It is also shown that the channel errors improve the behavior of bistable networks, and if the noise is strong enough, a network, otherwise bistable, becomes asymptotically stable. Thus channel errors, possibly introduced intentionally (and in such a manner that $A=I$ ), can be viewed as a decentralized stabilizing controller.

Acknowledgement-This work was supported by NSF Grant No. ECS 85-03031.

\section{REFERENCES}

1. J. L. Massey, Collision-Resolution Algorithms and Random-Access Communications. UCLA-ENG-8016, School of Engineering, and Applied Science University of California, Los Angeles, CA (1980).

2. G. S. Evseev and N. G. Ermolaev, Estimates of the Characteristics of Conflict Resolution in a Channel with Free Access and Noise. Probl. Peredachi Inf. 18, 101-105 (1982).

3. N. D. Vuedenskaya and B. S. Tsybakov, Random Multiple Access of Packets to a Channel with Errors. Probl. Peredachi Inf. 19, 52-68 (1983). 
4. J. T. Capetanakis. Tree Algorithms for Packet Broadcast Channels. IEEE Trans. Inf. Theory 25, 505-515 (1979).

5. J. Cicero, S. M. Meerkov and Z. Schuss. Large Homogeneous Communication Networks with Markovian Access Control I. Equilibria and Local Stability. J. Math. Anal. Appl. 103. 481-496 (1984).

6. J. Cicero, J.-T. Lim, S. M. Meerkov and Z. Schuss, Large Homogeneous Communication Networks with Markovian Access Control II. Global Behavior and Residence Time. J. Math. Anal. Appl. 105, 84-103 (1985).

7. J.-T. Lim, S. M. Meerkov and Z. Schuss State-Space Analysis of Static and Dynamic Markovian Access Control Protocols. Proceedings of the 23rd IEEE CDC, Las Vegas, NE, 1984, pp. 1364-1371.

8. S. M. Meerkov, Simplified Description of Slow Markov Walks, Par I. Auto. Remote Control 33, 404-414 (1972)

9. L. Ljung, Analysis of Recursive Stochastic Algorithms. IEEE Trans. Auto. Control 22, 551-575 (1977).

10. S. M. Meerkov and I. Rubin. System-Theoretic Properties of Large Communication Networks with Markovian Multiaccess Control Disçiplines. Syst. Control Lett. 1, 340-346 (1982)

11. B. Hajek, Hitting and Occupation Time Bounds Implied by Drift Analysis with Applications. Adv. Appl. Prob. 502 525 (1982)

12. P. R. S. Kumar and L. Merakos, Distributed Control of Broadcast Channels with Acknowledgement Feedback: Stability and Performance. Proceedings of the 23rd IEEE CDC, Las Vegas, NV, 1984, pp. 1143-1147. 